\title{
Global Warming Signals in Brazil and its Macro- Regions - Trend Analysis of Distributional Characteristics Characteristics
}

Jessica de Lima da Vida Pellenz ( $\sim$ jessipellenz@gmail.com )

Federal University of Vicosa: Universidade Federal de Vicosa https://orcid.org/0000-0001-7091-0964 Leonardo Bornacki de Mattos

Federal University of Vicosa: Universidade Federal de Vicosa

\section{Research Article}

Keywords: Global Warming, Brazil, Trend Test, Temperature.

Posted Date: December 21st, 2021

DOI: https://doi.org/10.21203/rs.3.rs-1179042/v1

License: (c) (1) This work is licensed under a Creative Commons Attribution 4.0 International License.

Read Full License 


\title{
Characteristics
}

June, 2021

\begin{abstract}
The aim of this research is to study global warming signals across Brazil. This investigation uses approximately 60 years of daily temperature data set and applies a recent trend test proposed by Rivas and Gonzalo (2020) which analyses not only the average but also different distributional characteristics. Besides, the test provides robust results for both $\mathrm{I}(0)$ and $\mathrm{I}(1)$ processes. We found significant trends in almost all characteristics in the analysis of the whole country. The mean and the maximum are increasing over time and the dispersion measures indicate decreasing trends. For the region analysis, we found out that, apart from the South, which does not appear to be drastically affected by global warming, the other regions present clear signs of global warming.
\end{abstract}

Keywords: Global Warming. Brazil. Trend Test. Temperature.

${ }^{*}$ Corresponding author - E-mail: jessipellenz@gmail.com 
 \\ 1 Introduction}

Climate Change issues, especially those related to Global Warming, are one of the most discussed matters nowadays. Increasing temperature and extreme climate events, regardless of their anthropogenic or natural origin, are perhaps the most challenging problem in modern society. Given the importance of this matter and the consequences it may bring, it is rather essential some basic characteristics inherent of the increasing process of climate variables.

To study Climate Change we must deeply understand four issues questions, which form a chain (IPCC. Intergovernmental Panel on Climate Change, 2014; Rivas and Gonzalo, 2020): (i) What type of Global Warming exists?; (ii) what are the causes of Global Warming (is it caused purely by human activities)?; (iii) what are the economic effects of Global Warming?; and (iv) what economic policies to mitigate these effects can be implemented?

If we consider the White and Granger (2011) definition, Global Warming is an evident increasing trend in some characteristics measuring the central tendency or position of the global temperature distribution. This definition is related to the first question mentioned by IPCC. So we notice the importance of understanding the trend in the temperature and in its distributional characteristics.

Many studies have been carried out to analyze the possible trends in temperature data as well as other climate variables over the years. While most of them have explored the temperature for the whole globe or for Europe, relatively fewer have been focused on Brazilian recorded temperature. Besides, considering the extension of Brazilian territory, and the heterogeneous characteristic of the regions, it is important to analyze each one of them to be sure that the possible effect of climate change is homogeneous throughout the country. Therefore, our objective with this paper is to try to understand the first question by analyzing the characteristics of temperature in Brazil.

The rest of the paper is organized as follows. In Section 2, we define Global Warming and the trends needed to investigate Global Warming, as well as the Rivas and Gonzalo (2020) approach for the trend test and the stationarity test. Section 3 provides the 
empirical applications: the whole country analysis, and the regional analysis. Finally, section 4 concludes the paper.

\section{Methodology and Data}

We performed our analysis according to Rivas and Gonzalo (2020) (hereafter RG) to identify Global Warming (GW) properties in Brazil from 1961 to 2019. To do so, we first introduce the GW definition which was synthesized by Rivas and Gonzalo (2020) according to the White and Granger (2011) propositions. For our purposes, we will evaluate the existence of trends in the statistical characteristics ${ }^{1}$ of the temperature distribution, and not only in its average values.

Definition 1 (Global Warming) $G W$ is an evident increasing trend in some characteristics measuring the central tendency or position of the global temperature distribution.

The tricky part of this paper is to determine a suitable definition of a trend. We consider the trend characterization first proposed by White and Granger (2011), in which two properties should be satisfied: (i) a trend should have a direction; and (ii) a trend should be somewhat smooth. We also include two other properties suggested by Rivas and Gonzalo (2020): (iii) a trend does not have to be monotonic throughout; and (iv) a trend can be a local behavior. These properties can be understood following the definitions of deterministic and stochastic trends.

\section{Definition 2 (Deterministic trend (White and Granger, 2011)) Consider $\left\{C_{t}\right\}=$} $\left\{C_{t}, t=0,1, \ldots, T\right\}$ as a sequence of real numbers. If $C_{t}<C_{t+1}$ for all $t$; then $\left\{C_{t}\right\}$ is an increasing trend. If $\left\{-C_{t}\right\}$ is an increasing trend, then $\left\{C_{t}\right\}$ is a decreasing trend.

If Definition 2 is only satisfied for a limited range, then $\left\{C_{t}\right\}$ is a (weak) increasing local trend.

\footnotetext{
${ }^{1}$ Such as minima and maxima, standard deviation, range, quantiles, etc.
} 
Definition 3 (Stochastic trend (White and Granger, 2011)) Let $X_{t}$ be a stochastic process determined as a function $X_{t}=f_{t}\left(Y_{t}, Y_{t-1}, \ldots\right)$.

1. Let $C_{t}=E\left(X_{t}\right)$ : if $C_{t}$ is a (weak) increasing trend, $X_{t}$ has a (weak) increasing trend in mean.

2. Let $C_{t}=\operatorname{var}\left(X_{t}\right)$ : if $C_{t}$ is a (weak) increasing trend, $X_{t}$ has a (weak) increasing trend in variance.

3. Let $C_{t}=E\left(\left[X_{t}-E\left(X_{t}\right)\right]^{k}\right)$ for finite positive integer $k$ : if $C_{t}$ is a (weak) increasing trend, $X_{t}$ has a (weak) increasing trend in kth central moment.

4. Let $C_{t}=E\left(\left[X_{t}-E\left(X_{t}\right)\right]^{k}\right)$ for finite positive real $k$ : If $C_{t}$ is a (weak) increasing trend, $X_{t}$ has a (weak) increasing trend in kth absolute central moment.

5. Let $C_{t}(p)=\inf \left\{x \in \Re: F_{t}(x) \geq p\right\}$ be the quantile $p \in(0,1)$ of the distribution function $F_{t}(x)=P\left(X_{t} \geq x\right)$. If $C_{t}(p)$ is a (weak) increasing trend, then $X_{t}$ has a (weak) increasing trend in quantile $p$.

Rivas and Gonzalo (2020) argue that from Definition 3, we can consider the stochastic trend the way it is defined by the econometrics literature as a deterministic trend in the second moment of the distribution. This is where the whole approach of the Rivas and Gonzalo (2020) trend test begins since they develop a method able to detect deterministic trends, and apply this method to different distributional characteristics.

\subsection{Trend detection (Rivas and Gonzalo, 2020)}

In order to fulfill some requirements of the trend test, we will consider that temperature is a functional stochastic process $X$ that must satisfy certain regularity conditions (the state densities and distribution) in order for the quantiles to be estimated consistently.

Definition 4 (Applying Definition 1) Let $h(t)$ be an increasing function oft. A characteristic $C_{t}$ of a stochastic process $X_{t}$ is said to contain a trend if the null hypothesis of $\beta=0$ is rejected in the regression 


$$
C_{t}=\alpha+\beta h(t)+u_{t}, \quad t=0,1, \ldots, T
$$

For the purpose of this paper, we use a linear trend to represent the function $h(t)$ in Equation (1). Then we can write (1) as

$$
C_{t}=\alpha+\beta t+u_{t}, \quad t=0,1, \ldots, T
$$

Rivas and Gonzalo (2020) highlights three important remarks referring to this definition: (i) the estimation of (1) has to be understood as the linear least squared approximation of an unknown function $h(t)$; (ii) the parâmeter $\beta$ is the plim of $\hat{\beta_{O L S}}$; (iii) in practice, in order to test $\beta=0$, it is recommended to use a robust HAC version of $t_{\beta=0}$. Therefore, in the empirical application, we estimate Equation (2) via OLS and implement the HAC version of $t_{\beta=0}$ as proposed by Newey and West (1986).

We also need the Definition 4 to satisfy the summability and Strength requirements so the $\beta$ have the right properties. The nexts two definitions guarantee that they are fulfilled.

Definition 5 (Order of Summability) A trend $h(t)$ is summable of order $\theta$, or $(S(\theta)$ ) if there exists a slowly-varying function $L(T)$, such that

$$
S_{T}=\frac{1}{T^{1+\theta}} L(T) \sum_{t=1}^{T} h(t)
$$

As we consider a linear function $h(t)=t($ then $h(t)>0)$ for the trend, we can rewrite the Equation (3) as:

$$
S_{T}=\frac{1}{T^{2}} \sum_{t=1}^{T} t=O p(1)
$$

therefore, $\theta=1$.

Definition 6 (Trend Strength) A trend function $h(t)$ is said to be stronger than another trend function $g(t)$ if $\theta_{h}>\theta_{g}$. 
Definitions 5 and 6 allow us to proceed with the RG trend test in a given characteristic $C_{t}=h(t)+I(0)$. First though, we have to call two propositions presented by Rivas and Gonzalo (2020).

Proposition 1 Let $C_{t}=I(0)$. In the OLS regression we have

$$
C_{t}=\alpha+\beta t+u_{t},
$$

The OLS estimator $\hat{\beta}$ satisfies

$$
T^{\frac{3}{2}} \hat{\beta}=O p(1)
$$

and asymptotically, $t_{\beta=0}$ is $N(0,1)$.

Proposition 2 Let $C_{t}=h(t)+I(0)$, such that $h(t)$ is an increasing $S(\theta)$ function with $\theta \geq 0$, and the function $g(t)=h(t) t$ is $S(\theta+1)$. In the OLS regression we have

$$
C_{t}=\alpha+\beta t+u_{t},
$$

The OLS estimator $\hat{\beta}$ satisfies

$$
T^{1-\theta} \hat{\beta}=O p(1)
$$

To proceed the behavioral analysis of the $t$-statistic $t_{\beta}=0$, we assume that the function $h(t)^{2}$ is $S(1+2 \theta \gamma)$, with $0 \leq \gamma \leq 1+\theta$. Then, the $t$-statistic diverges at the rates

$$
t_{\beta=0}\left\{\begin{array}{r}
O p\left(T^{\frac{\gamma}{2}} \hat{\beta}\right), \quad \text { for } 0 \leq \gamma \leq 1 \\
O p\left(T^{\frac{1}{2}} \hat{\beta}\right), \quad \text { for } 1 \leq \gamma \leq 1+\delta
\end{array}\right.
$$

In our case, $h(t)=t$, so the summability parameters are $\theta=1$ and $\gamma=1$. Then, in Equation (7), $\hat{\beta}=O p(1)$; but, $t_{\beta=0}$ diverges as $T \rightarrow \infty$.

Propositions 1 and 2 require the series to be stationary. What happens then, if some of the temperature characteristics are non-stationary $\left(C_{t}=I(1)\right)$ ? Rivas and Gonzalo 
(2020) say that in this case, $T^{\frac{1}{2}} \hat{\beta}=O p(1)$ and $t_{\beta}=0$ diverges as $T \rightarrow \infty$. Therefore, $\mathrm{RG}$ trend test can detect the stochastic trend generated also by $I(1)$ processes.

\section{$2.2 \quad$ Stationarity test}

There have been many studies debating the relevance of stationarity in climate research arguing that climate variables are constantly changing and as such, the mean and the variance of those variables can not be constant over time. Besides the intrinsic characteristics of the climate variables, also McCarl et al. (2008) argue that climate change could alter the distribution of stationarity and that is why we are analyzing it in this paper.

Most time series methods assume some kind of stationarity to guarantee efficient results. For example, if we face a variable that has the mean and variance volatile, it is impossible to make any trustable forecast or risk analysis because of the confidence interval is too high, which means we lost confidence in our predictions.

Our approach has no intention to make such assumptions, as we aim to verify the existence of climate change and not its implications in other variables. Even though, testing for stationarity has an important role in this analysis. As we mentioned before, stationarity can be altered by climate change, so we proceed with a stationarity test in order to make an initial guess about the existence of climate change in Brazil.

We proceed with the augmented Dickey-Fuller (ADF) test for unit root proposed by Dickey and Fuller (1979) and adapted by Said and Dickey (1984) in order to test for the presence of unit root (trend stationary). ADF tests are conducted through Ordinary least square estimation models including a linear trend. Beginning with the traditional Dickey-Fuller test, the ADF formulation allows the analysis to consider a general $A R(p, q)$ models with unknown orders. Consider an $A R(1)$ model:

$$
y_{t}=\beta t+\rho y_{t-1}+\epsilon_{t}, \quad t=0,1, \ldots, N
$$

where $y$ is the temperature characteristic at time $t, N$ is the number of observations, $\rho$ is the parameter to be estimated and $\epsilon$ is the error assumed to be white noise.If $|\rho| \geq 1, y_{t}$ 
163

is nonstationary and the variance of $y_{t}$ tends to infinity with time (explodes). If $|\rho|<1$, then $y_{t}$ is trend stationary. The hypothesis of trend stationarity can be evaluated by testing whether the absolute value of $\rho$ is strictly less than one. In ADF test the null hypothesis $H_{0}: \rho=1$ is tested against the one-sided alternative hypothesis. The standard DF test is carried out by estimating (10) after subtracting $y_{t-1}$ in both sides. Then it can be written as:

$$
\Delta y_{t}=\beta t+(\rho-1) y_{t-1}+\epsilon_{t},
$$

$$
\Delta y_{t}=\beta t+\delta y_{t-1}+\epsilon_{t}, t=0,1, \ldots, N
$$

where $\delta=\rho-1$. The null and alternative hypotheses are $H_{0}: \delta=0$ and $H_{1}: \delta \leq 0$. The maximum likelihood estimator of $\delta$ is denoted by $\hat{\delta}$ and calculated as follows:

$$
\hat{\delta}=\left(\sum_{t=2}^{N} y_{t-1}^{2}\right)^{-1} \sum_{t=2}^{N} y_{t} y_{t-1}
$$

The statistic for testing the null hypothesis that $\rho=1$ in (10), which is equivalent to $\delta=0$ in $(11 b)$, is based on the usual OLS (Ordinary Least Squares) t-test as:

$$
\hat{t}=\frac{\hat{\delta}-1}{\hat{\sigma}_{\hat{\delta}}}
$$

where $\hat{\sigma}_{\hat{\delta}}$ is the usual OLS standard error defined as:

$$
\hat{\sigma}_{\hat{\delta}}=S_{e}^{2}\left(\sum_{t=2}^{N} y_{t-1}^{2}\right)^{\frac{-1}{2}} \frac{S_{e}}{\sqrt{\sum_{t=2}^{N} y_{t-1}^{2}}}
$$

where $S_{e}$ denotes the standard deviation of the OLS estimate of the residuals in the regression model and it can be calculated as:

$$
S_{e}^{2}=\frac{1}{N-2} \sum_{t=2}^{N}\left(y_{t}^{2}-\hat{\delta} y_{t-1}\right)^{2}
$$


q) models with unknown orders. In this case, the test equation is given by:

$$
\Delta y_{t}=\beta t+\delta y_{t-1}+\sum_{j=1}^{p-1} \rho_{j+1} \Delta y_{t-j}+\epsilon_{t}
$$

The procedure follows the same steps as the standard DF presented above and $p$ is chosen by the Schwarz Information Criterion (Schwarz et al., 1978) ${ }^{2}$.

\subsection{Data set and characteristics calculation}

The temperature record series (thermometer measured) in Brazil run from 1961 to the present. These data are measured daily in 560 (actives and inactive) stations and it is provided by the Brazilian National Meteorological Institute (INMET). Figure 1 shows us the recorded mean temperature in Brazil over the period 1961-2019 measured daily, monthly and annually.
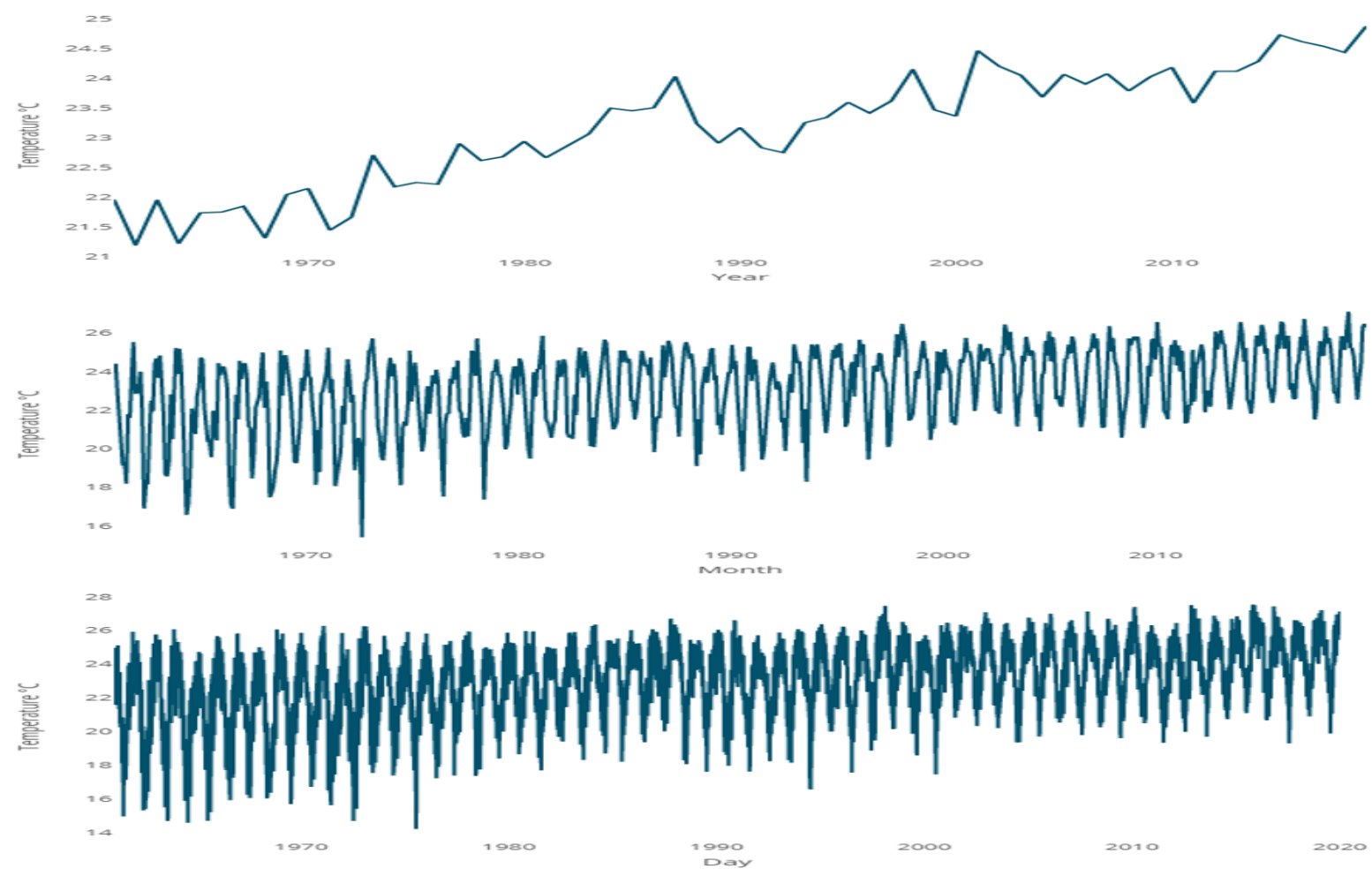

Figure 1: Recorded temperature data from INMET at different frequencies.

181 The advantages of the INMET database are its high frequency and the observational characteristic.In other words, the data does not suffer from bias due to the interpolation

\footnotetext{
${ }^{2}$ Same used in Rivas and Gonzalo (2020).
} 
or the inadequacy to approximate the calculation. Moreover, having daily observations for each year (1961-2019) allows us to calculate the distributional characteristics and convert them into time series objects. Figure 2 shows the path of these characteristics:

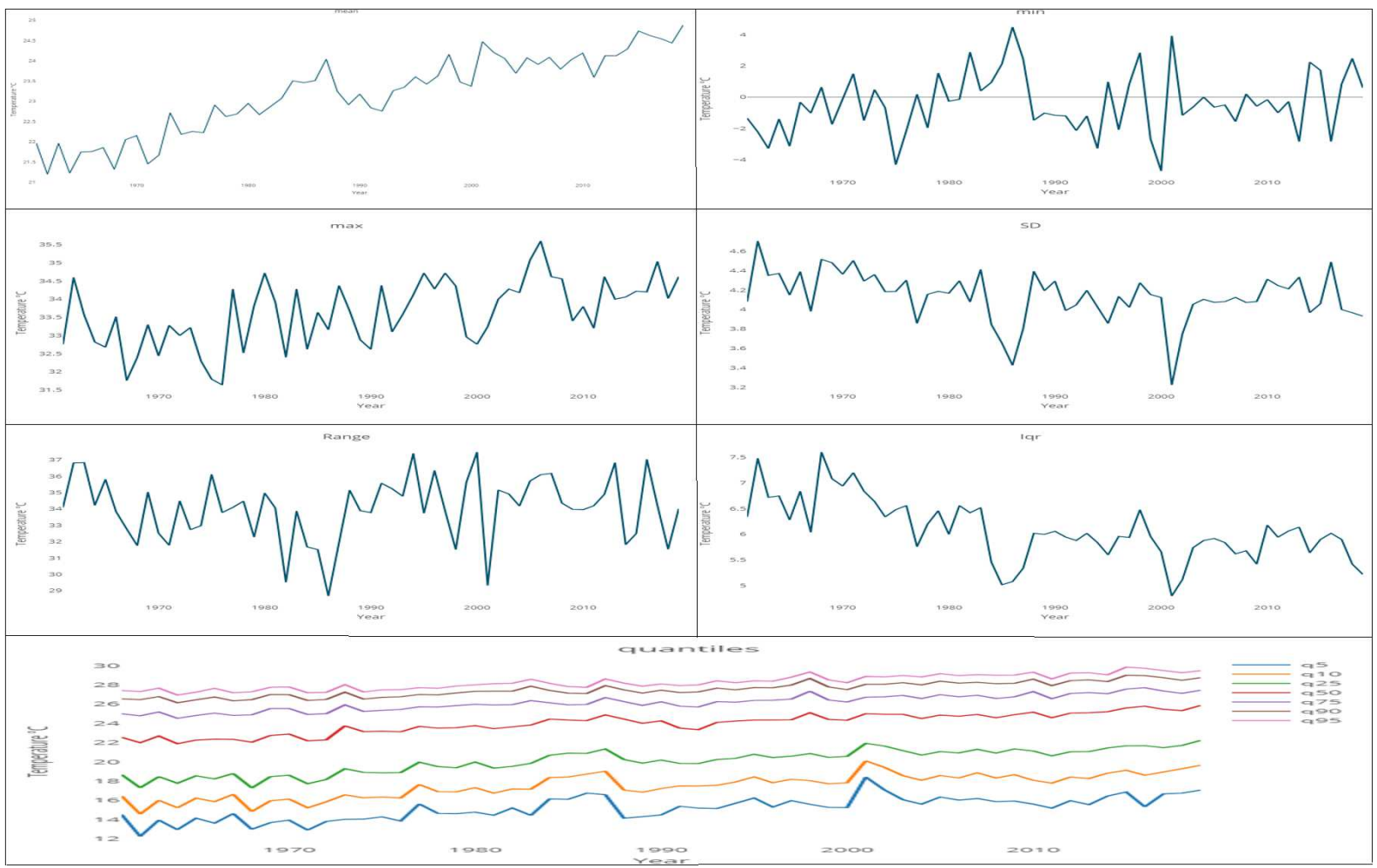

Figure 2: Annual distributional characteristics of temperature $\left({ }^{\circ} C\right)$ calculated with daily data.

\section{Results and Discussion}

In this section, we show the results for the ADF unit root and trend tests on the INMET daily temperature data first for 560 (active and inactive) Brazilian stations and then for each macro-region inside the country.

\subsection{Country Analysis}

Before we analyze the existence of trends in distributional characteristics of the temperature data to Brazil, we perform an ADF unit root test considering the SBIC criterion for lag selection. The variables tested were: mean, minimum (min), maximum $(\max )$, standard deviation (sd), interquantile range (iqr), range and 19 quatis (q5, q10, q15, q20, q25, q30, q35, q45, q50, q55, q60, q65, q70, q75, q80, q85, q90, q95). In the text, only 
the results of the quantiles q5, q25, q50, q75 and q95 are presented. Full results can be found in the appendix A.

The main results are presented in table 1 . We reject the null hypotheses of nonstationarity for the first six distributional characteristics (mean, min, max, sd, iqr, range), lowest quantiles (q5 to q15) and for higher quantiles (q80 to q95). Therefore, most of our quantiles (q20 to q75) are non-stationary. Rivas and Gonzalo (2020) argues that, even in the presence of non-stationarity, the trend test via OLS is able to provide reliable results about the existence of a trend in the series.

Table 1: Unit root rest (INMET data)

\begin{tabular}{lll}
\hline \hline Characteristic & ADF & Lags \\
\hline Mean & $-2.317^{* * *}$ & 3 \\
Min & $-5.098^{* * *}$ & 1 \\
Max & $-4.766^{* * *}$ & 1 \\
SD & $-4.243^{* * *}$ & 1 \\
Iqr & $-3.323^{*}$ & 1 \\
Range & $-2.955^{* *}$ & 3 \\
Q5 & $-4.114^{* *}$ & 1 \\
Q25 & -2.358 & 3 \\
Q50 & -2.358 & 3 \\
Q75 & -2.995 & 3 \\
Q95 & $-3.417^{*}$ & 3 \\
\hline
\end{tabular}

Source: Research results

Next, we apply the trend test to the distributional characteristics of Brazilian temperature. Table 2 reports the OLS trend coefficients and a HAC $t_{\beta}=0$ (significance level) and allows us to confirm the existence of a trend in most of the characteristics analyzed. The exception is the variables minimum and range, which did not show statistical significance for trend. We emphasize the similarity of the magnitudes of the quantile coefficients, especially some lower and medium ones (q10 to q45), which may show a unique trend 
for these variables. Subsequently, additional tests are carried out in order to prove this hypothesis.

Table 2: Trend test (INMET data)

\begin{tabular}{ll}
\hline \hline Characteristic & Coefficient \\
\hline Mean & $0.05363^{* * *}$ \\
Min & 0.01952 \\
Max & $0.02930^{* * *}$ \\
SD & $-0.00471^{* *}$ \\
Iqr & $-0.02028^{* * *}$ \\
Range & 0.00977 \\
Q5 & $0.05457^{* * *}$ \\
Q25 & $0.0647^{* * *}$ \\
Q50 & $0.05781^{* * *}$ \\
Q75 & $0.04441^{* * *}$ \\
Q95 & $0.04063^{* * *}$ \\
\hline
\end{tabular}

Source: Research results

Table 2 shows us that all trend coefficients are positive, except for the dispersion measures (standard error and interquartile range) which are negative. We can see that the mean has a coefficient of 0.05 , which implies an increase of 2.95 degrees Celsius in 59 years. This result is ratified by the figure 1 which shows the temperature increasing from 22 degrees Celsius in 1961 to nearly 25 degrees in 2019.

The negative coefficients for the standard deviation indicate that the country is facing temperatures closer to the mean as the years go by. Also, as the mean temperature is increasing, we can say that the temperature is less volatile around an increasingly average. Furthermore, the trend coefficients of the lower quantiles presented higher magnitude than the upper ones, indicating that the temperature is decreasing its dispersion.

In order to highlight the importance of the quantiles analysis, we performed auxiliary tests to verify whether the dispersion between the quantiles are decreasing or not. We 
also estimate the Wald test to check for equality in trend coefficients. Table 3 shows the results.

Table 3: Co-trending and spacing analysis (INMET data)

\begin{tabular}{ll}
\hline \hline Characteristic & Wald test \\
\hline All quantiles (q5, q10, .., q95) & $15.79^{* * *}$ \\
Lower quantiles (q5-q25) & $11.12^{* * *}$ \\
Medium quantiles (q50-q75) & $57.13^{* * *}$ \\
Upper quantiles (q90-q95) & 1.67 \\
\hline \hline Spacing & \\
\hline Characteristic & Trend coefficient \\
\hline q50-q5 & 0.003236 \\
q95-q50 & $-0.017178^{* * *}$ \\
q95-q5 & $-0.013942^{* *}$ \\
\hline
\end{tabular}

Source: Research results

As we can see in table 3, the Wald test guides us to reject the null hypotheses of equal coefficients for all the quantiles as well as for the lower and medium quantiles. Although, this is not the case if we test the null hypothesis of equal trends in the upper quantiles, since we can not reject that the coefficients are the same for these variables. The trend test on the spacing characteristics reveled the existence of a decreasing trend between the fourth quartile (q95) and the median (q50). Nevertheless, the difference between the median and the lowest quantile (q5) does not show a statistically significant trend.

Results from 3 infer that the distance between the mean and maximum temperature are reducing. In other words, the mean temperature in Brazil is rising as the time passes by, confirming once more the results in 2 . These results also imply that the findings as in Rivas and Gonzalo (2020) and IPCC. Intergovernmental Panel on Climate Change (2014) reporting that winters warmed more than summers do not apply to Brazil, since minimum temperatures, in general, do not show an upward trend and the spacing test between lower and middle quantiles presents no statistical significance. 
As discussed in the introduction, Brazil is a country with continental proportions, therefore, its climate is not homogeneous in all regions. Temperature discrepancies vary considerably from the north (where a warm climate prevails throughout the year) to the south (which has four well-defined seasons reaching quite low temperatures, especially during the winter). In order to contemplate this heterogeneity, in the following section, we conduct the unit root and trend test for each region.

\subsection{Macro-region Analysis}

The ADF test for the regions revealed even more non-stationary distributional characteristics than in the country analysis except for the south, where we can reject the null hypotheses of non-stationarity in all variables. The north is the region with the most non-stationary variables. The main results to ADF test are displayed in table 4.

Table 4: Region unit root test (INMET data)

\begin{tabular}{l|ll|ll|ll|ll|ll}
\hline \multicolumn{3}{c}{ N } & \multicolumn{3}{c}{ NE } & \multicolumn{3}{c}{ SE } & \multicolumn{2}{c}{ MW } \\
\hline Charac & ADF & Lags & ADF & Lags & ADF & Lags & ADF & Lags & ADF & Lags \\
\hline Mean & $-3.64^{* *}$ & 3 & $-3.52^{* *}$ & 4 & -3.07 & 4 & $-4.53^{* * *}$ & 3 & $-5.43^{* * *}$ & 1 \\
Min & $-6.06^{* * *}$ & 1 & $-4.01^{* *}$ & 1 & $-4.38^{* * *}$ & 1 & $-5.01^{* * *}$ & 1 & $-5.07^{* * *}$ & 1 \\
Max & -0.87 & 7 & $-4.67^{* * *}$ & 2 & $-5.20^{* * *}$ & 1 & $-4.57^{* * *}$ & 1 & $-4.80^{* * *}$ & 1 \\
SD & $-5.23^{* * *}$ & 2 & $-4.43^{* * *}$ & 1 & $-4.54^{* * *}$ & 1 & $-5.25^{* * *}$ & 1 & $-6.14^{* * *}$ & 1 \\
Iqr & -2.58 & 4 & $-4.15^{* * *}$ & 1 & $-4.88^{* * *}$ & 1 & $-3.88^{* * *}$ & 1 & $-5.76^{* * *}$ & 1 \\
Range & $-6.59^{* * *}$ & 1 & $-3.27^{*}$ & 2 & $-3.73^{* *}$ & 2 & $-5.74^{* * *}$ & 1 & $-4.65^{* * *}$ & 1 \\
Q5 & $-3.76^{* *}$ & 4 & $-3.23^{*}$ & 4 & $-5.51^{* * *}$ & 1 & $-3.32^{*}$ & 5 & $-6.70^{* * *}$ & 1 \\
Q25 & $-3.35^{*}$ & 4 & -2.70 & 4 & -3.15 & 4 & $-4.01^{* *}$ & 3 & $-5.98^{* * *}$ & 1 \\
Q50 & $-3.64^{* *}$ & 4 & $-3.37^{*}$ & 4 & -2.75 & 3 & $-4.85^{* * *}$ & 3 & $-5.34^{* * *}$ & 1 \\
Q75 & $-3.43^{*}$ & 4 & $-4.17^{* * *}$ & 3 & -3.03 & 3 & $-4.74^{* * *}$ & 3 & $-3.84^{* * *}$ & 1 \\
Q95 & $-3.32^{*}$ & 3 & $-5.74^{* * *}$ & 1 & $-4.49^{* * *}$ & 1 & $-4.86^{* * *}$ & 3 & $-2.85^{* * *}$ & 2 \\
\hline
\end{tabular}

Source: Research results

Note: N, NE, SE, MW and S are the regions North, Northeast, Southeast, Midwest and South.

We also conducted the trend test for each Brazilian region. The trend coefficients and the $\mathrm{HAC} t_{\beta}=0$ (significance level) are presented in table 5 . We can see that in the 
Table 5: Region trend test (INMET data)

\begin{tabular}{l|l|l|l|l|l}
\hline \multicolumn{5}{c}{ Coefficients } \\
\hline Charac & $\mathrm{N}$ & $\mathrm{NE}$ & $\mathrm{SE}$ & $\mathrm{MW}$ & $\mathrm{S}$ \\
\hline Mean & $0.0322^{* * *}$ & $0.0342^{* * *}$ & $0.0321^{* * *}$ & $0.0296^{* * *}$ & $0.0079^{* *}$ \\
Min & -0.0105 & -0.0195 & $0.0884^{* * *}$ & $0.0696^{* * *}$ & 0.0194 \\
Max & $0.0522^{* * *}$ & $0.0454^{* * *}$ & $0.0195^{* * *}$ & $0.0240^{* * *}$ & 0.0098 \\
SD & $0.0027^{* * *}$ & $0.0019^{* *}$ & $-0.0034^{* *}$ & $-0.0085^{* * *}$ & $0.0033^{*}$ \\
Iqr & $0.0066^{* * *}$ & -0.0022 & $-0.0066^{* *}$ & $-0.0136^{* * *}$ & $0.0079^{*}$ \\
Range & $0.0627^{* * *}$ & $0.0648^{* * *}$ & $-0.0689^{* * *}$ & $0.0416^{* * *}$ & -0.0096 \\
Q5 & $0.0297^{* * *}$ & $0.027^{* * *}$ & $0.033^{* * *}$ & $0.04162^{* * *}$ & 0.00093 \\
Q25 & $0.0287^{* * *}$ & $0.0358^{* * *}$ & $0.0360^{* * *}$ & $0.0363^{* * *}$ & 0.0032 \\
Q50 & $0.0316^{* * *}$ & $0.0354^{* * *}$ & $0.0333^{* * *}$ & $0.0281^{* * *}$ & $0.0101^{* * *}$ \\
Q75 & $0.0353^{* * *}$ & $0.0336^{* * *}$ & $0.0294^{* * *}$ & $0.0227^{* * *}$ & $0.0111^{* * *}$ \\
Q95 & $0.0361^{* * *}$ & $0.0364^{* * *}$ & $0.0254^{* * *}$ & $0.0199^{* * *}$ & $0.0104^{* * *}$ \\
\hline
\end{tabular}

Source: Research results

Note: N, NE, SE, MW and S are the regions North, Northeast, Southeast, Midwest and South.

Southern region, where the climate is relatively milder than in the rest of the country, only the average and the quantiles above the q40 have significant trend coefficients (at the level of 5\%), which means that, in this region, lower temperatures do not present an increasing trend. 
North and Northeast, which presented larger magnitudes in the coefficients than in other regions.

Another important finding refers to the Southeast and Midwest, two important agribusiness regions. They show larger coefficient magnitudes in minimum temperature added to the lower quantiles coefficient relatively higher than the upper ones. This means that the lower temperatures are approaching the mean more rapidly than otherwise. Besides, the negative sign in the standard deviation coefficient implies a reduced variation in the temperature, which is increasing.

In order to have more detailed results about the dispersion and spacing characteristics, we implemented the Wald test for the quantiles coefficients and the trend test for the difference between quantiles. The results are presented in table 6 .

Table 6: Co-trending and spacing analysis (INMET data)

\begin{tabular}{l|l|l|l|l|l}
\hline \hline \multicolumn{7}{c}{ Wald test statistics } \\
\hline Charac & $\mathrm{N}$ & $\mathrm{NE}$ & $\mathrm{SE}$ & $\mathrm{MW}$ & $\mathrm{S}$ \\
\hline All quantiles (q5, q1, .., q95) & $18.89^{* * *}$ & $12.02^{* * *}$ & $5.54^{* * *}$ & $6.44^{* * *}$ & 1.28 \\
Lower quantiles (q5-q25) & 1.04 & $21.9^{* * *}$ & 1.65 & $3.58^{* *}$ & 0.20 \\
Medium quantiles (q50-q75) & $80.56^{* * *}$ & $3.58^{*}$ & $14.32^{* * *}$ & $31.5^{* * *}$ & 0.18 \\
Upper quantiles (q90-q95) & 0.66 & 1.43 & $4.81^{* *}$ & 0.6 & 0.05 \\
\hline \hline & $\mathrm{Spacing}-$ Trend coefficients & & \\
\hline Charac & $\mathrm{N}$ & $\mathrm{NE}$ & $\mathrm{SE}$ & $\mathrm{MW}$ & $\mathrm{S}$ \\
\hline q50-q5 & $0.0025^{* *}$ & $0.0083^{* * *}$ & -0.0002 & $-0.0135^{* * *}$ & 0.0092 \\
q95-q50 & $0.0045^{* * *}$ & 0.0010 & $-0.0079^{* * *}$ & $-0.0083^{* * *}$ & 0.0003 \\
q95-q5 & $0.0070^{* * *}$ & $0.0093^{* * *}$ & $-0.0081^{*}$ & $-0.0218^{* * *}$ & 0.0095 \\
\hline
\end{tabular}

Source: Research results

Note: N, NE, SE, MW and S are the regions North, Northeast, Southeast, Midwest and South.

The Wald test for the South shows that we can not reject the null hypothesis of equal quantiles coefficients in all cases. So, we can infer that, if there is a trend in those characteristics, then they are all equal. The same does not apply to other regions. For the spacing analysis, we verified some important implications, especially for the Midwest 
region. In this region, the spacing coefficients are negative and statistically significant, revealing a reduction in the temperature variation around the average. Furthermore, the magnitude of the coefficient of q50-q5 is greater than that of q95-q50, which reveals that lower temperatures are closing up to the average faster than the high temperatures are. These results show convergence with the findings of Rivas and Gonzalo (2020) for trends in global temperature.

\section{Conclusion}

This study applies the Rivas and Gonzalo (2020) approach to modeling the evolution of certain temperature distributional characteristics. The test consists of estimating OLS regressions for each characteristic in order to reveal the trend coefficients. We proceeded with the test using the temperature record series for Brazil from 1961 to 2019 provided by the Brazilian National Meteorological Institute (INMET).

The analysis for the whole country shows a significant and positive trend for the mean, maximum, and for all quantiles. These results reveal a higher range of temperatures since the minimum does not change while the maximum ones tend to increase. The dispersion measures (standard error and interquartile range) are significant and negative. This result is confirmed by co-trending and spacing tests, which show a tendency towards a reduction in temperature dispersion related to the average values, especially pointing a smaller distance from the mean towards higher temperatures.

Although there has been no evidence of an increasing trend in minimum temperatures in the country's analysis, there is clear evidence global warming signs with regard to average and maximum temperatures and the behavior of dispersion and spacing variables. Considering the Brazilian territorial extension, tests were also carried out for each region of the country, aiming to separate the effects of different types of climate and characteristics of each macro-region.

The separate analysis for the Brazilian regions points to statistically significant trends in almost all distribution characteristics in all regions except for the South, where it seems to be barely affected by global warming. Apart from the South, the mean, maximum, 
and all quantiles seem to be increasing over time, which reveals that temperatures are increasing systematically over the years. In other words, the closer to the equator line, the more likely it is to feel the signs of Global Warming.

The most affected regions by Global Warming effects seem to be the Southeast and the Midwest, that show an increase in mean, minimum and maximum temperatures and, in addition, they present less favorable dispersion and spacing characteristics. The north and northeast also show significant trends in most distributional characteristics, except for minimum temperatures, which do not appear to be changing.

\section{Acknowedgments}

This study was financed in part by the Coordenação de Aperfeiçoamento de Pessoal de Nível Superior - Brasil (CAPES) - Finance Code 001.

\section{Data Availability}

The data used to support the findings of this study were taken from the Brazilian National Institute of Meteorology (INMET) ${ }^{3}$.

\footnotetext{
${ }^{3}$ Available online at: https://bdmep.inmet.gov.br/
} 
${ }_{322}$ Appendix A - Complete Results

Table 7: Country Analysis - Unit root rest (INMET data)

\begin{tabular}{|c|c|c|}
\hline Characteristic & $\mathrm{ADF}$ & Lags \\
\hline Mean & $-2.317^{* * *}$ & 3 \\
\hline Min & $-5.098^{* * *}$ & 1 \\
\hline $\operatorname{Max}$ & $-4.766^{* * *}$ & 1 \\
\hline SD & $-4.243^{* * *}$ & 1 \\
\hline Iqr & $-3.458^{* *}$ & 1 \\
\hline Range & -2.955 & 3 \\
\hline Q5 & $-4.114^{* *}$ & 1 \\
\hline Q10 & $-3,78^{* *}$ & 1 \\
\hline Q15 & $-3.512^{* *}$ & 1 \\
\hline Q20 & -2.431 & 3 \\
\hline Q25 & -2.35 & 3 \\
\hline Q30 & -2.390 & 3 \\
\hline Q35 & -2.419 & 3 \\
\hline Q40 & -2.396 & 3 \\
\hline Q45 & -2.385 & 3 \\
\hline Q50 & -2.358 & 3 \\
\hline Q55 & -2.390 & 3 \\
\hline Q60 & -2.462 & 3 \\
\hline Q65 & -2.604 & 3 \\
\hline Q70 & -2.794 & 3 \\
\hline Q75 & -2.995 & 3 \\
\hline Q80 & $-3.274^{*}$ & 3 \\
\hline Q85 & $-3.403^{* *}$ & 3 \\
\hline Q90 & $-3.480^{* *}$ & 3 \\
\hline Q95 & $-3.417^{*}$ & 3 \\
\hline
\end{tabular}

Source: Research results 
Table 8: Country Analysis - Trend test (INMET data)

\begin{tabular}{ll}
\hline \hline Characteristic & Coefficient \\
\hline Mean & $0.05363^{* * *}$ \\
Min & 0.01952 \\
Max & $0.02930^{* * *}$ \\
SD & $-0.00471^{* *}$ \\
Iqr & $-0.02170^{* * *}$ \\
Range & 0.00977 \\
Q5 & $0.05457^{* * *}$ \\
Q10 & $0.06239^{* * *}$ \\
Q15 & $0.06642^{* * *}$ \\
Q20 & $0.06611^{* * *}$ \\
Q25 & $0.0647^{* * *}$ \\
Q30 & $0.06338^{* * *}$ \\
Q35 & $0.06249^{* * *}$ \\
Q40 & $0.06156^{* * *}$ \\
Q45 & $0.06012^{* * *}$ \\
Q50 & $0.05781^{* * *}$ \\
Q55 & $0.0551^{* * *}$ \\
Q60 & $0.05203^{* * *}$ \\
Q65 & $0.04916^{* * *}$ \\
Q70 & $0.04662^{* * *}$ \\
Q75 & $0.04441^{* * *}$ \\
Q80 & $0.04292^{* * *}$ \\
Q85 & $0.04176^{* * *}$ \\
Q90 & $0.04122^{* * *}$ \\
\hline So & $0.043^{* * *}$ \\
\hline
\end{tabular}

Source: Research results 


\section{${ }_{323}$ References}

Dickey, D.A., Fuller, W.A., 1979. Distribution of the estimators for autoregressive time series with a unit root. Journal of the American statistical association 74, 427-431.

IPCC. Intergovernmental Panel on Climate Change, 2014. Synthesis report. Contribution of working groups I, II and III to the fifth assessment report of the intergovernmental panel on climate change. Technical Report. Geneva, Switzerland.

McCarl, B., Villavicencio, X., wu, X., 2008. Climate change and future analysis: Is stationarity dying? American Journal of Agricultural Economics 90, 1241-1247. doi:10. $1111 / j .1467-8276.2008 .01211 . x$

Newey, W.K., West, K.D., 1986. A simple, positive semi-definite, heteroskedasticity and autocorrelation consistent covariance matrix. Technical Report. National Bureau of Economic Research.

Rivas, M.D.G., Gonzalo, J., 2020. Trends in distributional characteristics: Existence of global warming. Journal of Econometrics 214, 153-174.

Said, S.E., Dickey, D.A., 1984. Testing for unit roots in autoregressive-moving average models of unknown order. Biometrika 71, 599-607.

Schwarz, G., et al., 1978. Estimating the dimension of a model. The annals of statistics $6,461-464$.

White, H., Granger, C.W., 2011. Consideration of Trends in Time Series. Journal of Time Series Econometrics 3, 1-40. 